\title{
The soft touch of robots
}

Among the most famous robots are the beloved Star Wars characters R2D2 and C3PO, who are humanoid robots (almost) perfectly capable of interacting with humans. In the real world, robots now have key roles in industry and medicine, and the field of robotics is expected to play a major part in the 4 th industrial revolution. We have come a long way from giant industrial robots to tiny machines and devices that can help in surgery. However, like R2D2 and C3PO, most current robots are made of rigid components with pre-programmed tasks, making their application problematic once they operate in close contact with or even in humans, or if they need to autonomously navigate in complex environments, such as space or water.

Soft robotics is an emerging field aiming to fabricate soft robots for close machine-human interactions. The soft nature allows the robots to adapt to their surroundings, to perform different and even autonomous

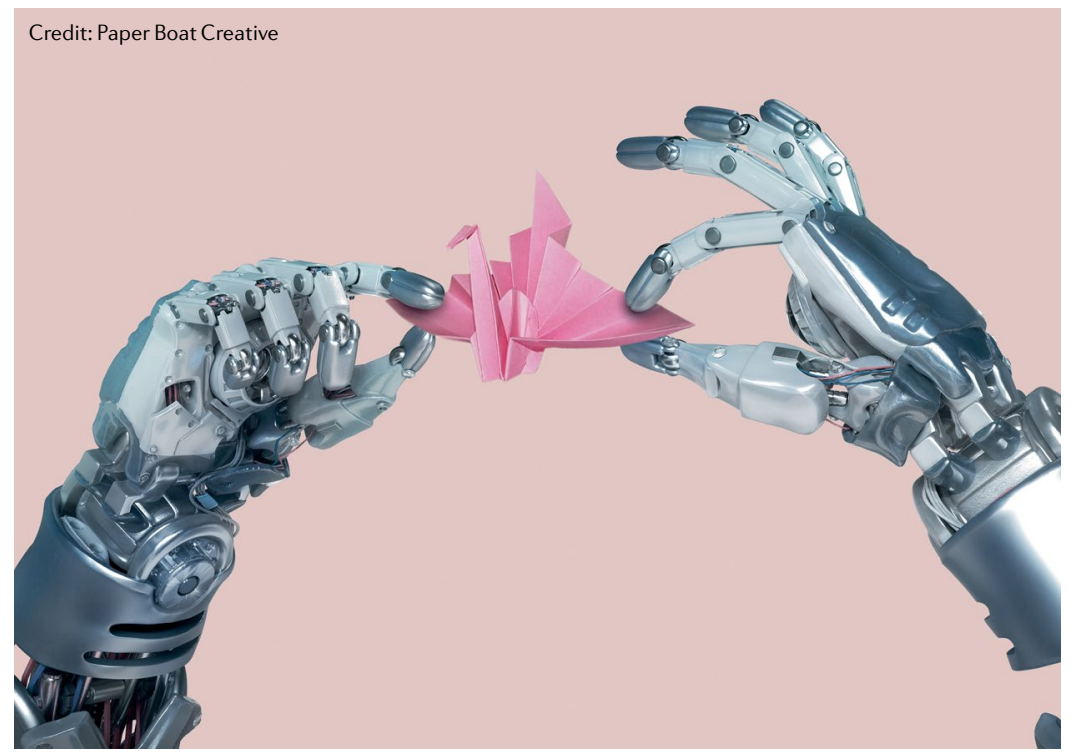

tasks, and to mimic the motion and function of biological systems, such as human muscles or the locomotion of bacterial cells. The field strives to mimic nature by designing biology-inspired structures and by recreating the way humans, animals and cells move. Soft and smart materials, such as ionotronic hydrogels and shape-memory polymers, are important in the design of soft robots and their chemistry and manufacturing are at the centre of current research efforts. Fabrication technologies, such as 3D printing and origami folding, have enabled the engineering of robots based on soft materials and have opened opportunities for the implementation of autonomic behaviours and sophisticated performance.

Combined efforts of materials science, computational design and creative engineering have already led to the development of origami robots that unfold and function once they reach a specific environment, of small soft robots that can navigate in biological tissue to excise cells or deliver drugs, of inflatable robots that can exert force to pick up objects and of exoskeletons and prostheses that can be worn to support or replace the function of human muscles.

The ultimate goal is the integration of soft actuators, sensors, controls and power systems without any hard components using a single, low-cost fabrication process to design fully artificial, intelligent soft robots that can function as medical, assistive and wearable devices, that can work in space or within a human organ, that can replace muscles or even whole organs and that are capable to safely interact with humans.

A number of challenges remain in the materials, engineering and design aspects of soft robots, but the great variety in soft materials, fabrication, design technologies and real-world applications of soft robots discussed in this Focus issue demonstrate that the stage is already set for a soft robotic future. We hope you enjoy reading these Research Highlights, Comments and Review articles on this topic. 